\title{
VI. Politische Säuberung
}

Neben den Prozessen gegen die Hauptschuldigen bildeten die breiter angelegte Entnazifizierung bzw. Entmilitarisierung der (west-)deutschen und der japanischen Bevölkerung ein Kernstück der amerikanischen Besatzungspolitik. Dabei stand in beiden Fällen die Überzeugung Pate, daß nur eine tiefgreifende Transformation der gesellschaftlichen Strukturen einschließlich einer Ausschaltung der sie bislang tragenden Schichten eine zukünftige Bedrohung des Weltfriedens durch die Aggressorstaaten des Zweiten Weltkrieges verhindern, die besiegten Länder langfristig pazifizieren, innerlich stabilisieren und ihnen einen demokratischen Weg nach amerikanischem Verständnis ermöglichen könnte. Dem deutschen wie dem japanischen Volk begegnete die führende westliche Siegermacht also mit einem durch den Kriegsausgang noch gestärkten Vertrauen in die Überlegenheit des eigenen politischen Systems, das an Woodrow Wilsons Credo „make the world safe for democracy“ anknüpfte ${ }^{1}$. Während man allerdings dem kulturell eng verwandten Deutschland, gestützt auf die Weimarer Erfahrungen und kontrolliert durch die Macht einer direkten Militärverwaltung, den Aufbau einer dezentralen Demokratie zutraute, war in bezug auf Japan die Einschätzung verbreitet, „es zu vermasseln“, wenn man versuchen würde, die deutschlandpolitischen Besatzungsrezepte eins zu eins auf den Inselstaat zu übertragen: „Die Japaner sind ein orientalisches Volk mit orientalischer Gesinnung und Religion."2 Aus dem Urteil über Konsenszwang und feudale Gefolgschaftstreue als tragende Elemente der nationalen politischen Kultur resultierten Vorstellungen von der Entwicklung einer „Demokratie auf japanisch“, die wesentlich unspezifischer blieben als für Deutschland und die vor allem auch Folgen für den politischen Säuberungsprozeß zeitigen sollten ${ }^{3}$.

In dieser Hinsicht schien die Aufgabe der USA in Japan insofern leichter, weil sie dort nicht mit drei weiteren Besatzungsmächten zu kooperieren hatten und zudem eine funktionierende Bürokratie zur Identifizierung und Auffindung der zu Säubernden heranziehen konnten. Zudem mußte die vor allem als „Entmilitarisierung“ verstandene Aktion vermutlich ein geringeres Ausmaß annehmen; hatten doch die grausamen Bilder bei der Befreiung der

1 Gasteyger, Das außenpolitische Erbe, S. 169.

2 So der US-Verteidigungsminister Henry L. Stimson, zit. bei Buckley, Japan Today, S. 8.

3 Rosenzweig, Erziehung, S. 108. 
nationalsozialistischen Konzentrationslager zumindest kurzzeitig dazu geführt, „that the Japanese were regarded as bad, but the Germans as downright evil" 4 . Erschwerend wirkte sich demgegenüber aus, daß von großen Teilen der japanischen Verwaltungselite paradoxerweise erwartet wurde, „zugleich das Land und sich selbst zu demokratisieren und zu säubern“5. Zudem hatte es in Japan - anders als im Dritten Reich mit der nationalsozialistischen Massenbewegung samt ihren Unterorganisationen - nicht eine einzige, säuberungspolitisch leicht faßbare Staatspartei gegeben, sondern neben dem Bund zur Förderung der kaiserlichen Herrschaft und unterschiedlich stark „faschistoid“ eingefärbten Parteien eine ganze Reihe von „ultranationalistischen, terroristischen und militaristischen Gruppen“. Im Vollzug der Direktive des SCAP vom 4. Januar 1946 waren so bis zum Ende der Besatzungszeit über 200 derartige Organisationen aufzulösen ${ }^{6}$.

Die als „Direktive vom 4. Januar“ bekannt gewordene SCAP-Anweisung (Nr. 550) implementierte eine Anordnung der Joint Chiefs of Staff, wonach sicherzustellen war, daß „aktive Vertreter des militanten Nationalismus“ und des Angriffskriegs oder solche, die den Zielen der Besatzungspolitik feindlich gegenüberstünden, aus dem öffentlichen Dienst oder anderen verantwortungsvollen und einflußreichen Positionen - einschließlich des besonders erwähnten Wirtschafts- und Finanzsektors - zu entfernen und auszuschließen seien. Bis zum Beweis des Gegenteils sollte generell davon ausgegangen werden, daß Personen, die in den Jahren seit 1937 Schlüsselpositionen innehatten, zum Kreis der Schuldigen zählten. Dabei unterschied SCAP zwischen sieben Kategorien bzw. Gruppen (A bis G): Kriegsverbrecher (A), Berufsoffiziere cinschließlich höheren Personals im Kriegsministerium oder in Militärpolizei und Geheimdiensten (B), führende Mitglieder radikaler nationalistischer Organisationen (C), einflußreiche Personen des Bundes zur Förderung der Kaiserlichen Herrschaft und des Politischen Vereins Großjapans (Dai Nippon Seiji-Kai) (D), Verwaltungsratsmitglieder von Finanz- und Wirtschaftsgesellschaften, die als Manager in den besetzten Gebieten „in die japanische Expansion involviert“ waren (E), Zivil- und Militärgouverneure im japanischen „Kolonialreich der Kriegszeit“ $(F)$ sowie „sonstige Militaristen und Ultranationalisten“ (G), das hieß Denunzianten, die zur Verhaftung oder Mißhandlung japanischer Oppositioneller beigetragen hatten, aber auch sämtliche Personen, die auf der nationalen Regierungsebene am japanischen Expansionsprogramm beteiligt waren und sich

4 Anthon, Reeducation for Democracy, S. 261. Schon bald aber erreichte das Japan-Feindbild in den USA, das auch während des Krieges deutlich stärker „von Haß-Propaganda geprägt“ gewesen war, wieder seine - aus rassistisch-kulturchauvinistischen Motiven heraus - traditionell schärfere Ausprägung. Bereits im Juni 1945 meinten 82\% der an eincr Gallup-Umfrage beteiligten Amerikaner, das japanische Volk sei im Vergleich zum deutschen „more cruel at heart". Eckert, Feindbilder im Wandel, S. 157, 161.

5 Livingston/Moore/Oldfather (Hg.), The Japan Reader 2: Postwar Japan, S. 4.

6 Seifert, Nationalismus im Nachkriegs-Japan, S. 113. 
in Reden, Schriften oder Taten als ,aktive Verfechter des militanten Nationalismus und Angriffskriegs" erwiesen hatten”.

Die Wirkung dieser ersten Säuberungsdirektive, deren Kategorien von der japanischen Regierung in einigen Punkten noch präzisiert wurden, ließ nicht lange auf sich warten. 20000 Personen suchten von sich aus rasch um Entlassung nach, um nicht als zu Säubernde designiert zu werden. Auch das amtierende Kabinett Shidehara mußte neun Tage nach Veröffentlichung der Direktive vom 4. Januar 1946 umgebildet werden, weil drei Minister und eine Reihe weiterer hochrangiger Regierungsbeamter ihr Ȧmt niederlegten. Als am 10. März Ausführungsbestimmungen zu der „catchall“-Kategorie G angekündigt wurden, verließen weitere fünf Minister das Kabinett, zahlreiche Mitglieder des Oberhauses folgten ihnen, Parteiführungen wechselten, alte Loyalitäten zerbrachen, die „Progressive Partei“ sah sich nahezu ausgelöscht, kurz: die politische Landschaft wurde umgepflügt. Auch von den 3384 nominierten Kandidaten für die bevorstehenden Parlamentswahlen im April 1946 fielen 252 unter die Bestimmungen der Säuberungsdirektive, die knappe Hälfte davon, weil sie bei den Kriegswahlen 1942 von Premierminister Tôjô empfohlen worden war. Die gewählten Abgeordneten wurden dann einer erneuten Prüfung unterzogen, in deren Folge die japanische Regierung neun Politikern das Mandat wieder entzog. Darüber hinaus fiel der prominente Vorsitzende der Liberalen Partei, Hatoyama Ichiro, dem Eingreifen der SCAP zum Opfer. Zahlenmäßig am stärksten von dieser ersten, bis August 1946 dauernden Säuberungswelle waren aber nicht Politiker betroffen, sondern Personen der Kategorie B (Berufsoffiziere etc.), von denen 183000 aus dem Öffentlichen Dienst ausgeschlossen wurden ${ }^{8}$. In den anderen Gruppen waren zusammengenommen 5520 Fälle überprüft worden, wovon aber nur 814 Verfahren negativ endeten.

Die niedrige Zahl an Entlassungen war den amerikanischen Behörden im Laufe des Jahres 1946 mehr und mehr ein Dorn im Auge. In der NiigataPräfektur hatte man etwa von 13000 überprüften Lehrern nur einen einzigen ausgeschlossen. Und zur Arbeit des Untersuchungskomitees in der Provinz Gumma bemerkte das zuständige Military Government Team: "The committee is screening, little fish with a big net " "9. Nun waren die Lehrer im japanischen Erziehungssystem tatsächlich einer strikten hierarchischen Kontrolle unterworfen gewesen und hatten nur selten Eigeninitiative bei der Propagierung ultranationalistischer Ziele entfalten können bzw. zu brauchen, aber auch auf Universitätsebene verlief die Säuberung durch die japanischen Behörden derart zurückhaltend, daß Studenten oder kritische Bürger sich immer wieder veranlaßt sahen, gegen Fehlentscheidungen

9 Rosenzweig, Erziehung, S. 124. 
vorzugehen. Der Kontrolle durch amerikanische Stellen waren nicht zuletzt durch die Sprachbarriere Grenzen gesetzt, da eine Übersetzung der ausgefüllten Fragebögen ins Englische aus Zeitnot und Personalmangel häufig gar nicht möglich war. Um so gravierender mußte sich dies auswirken, weil bei der Auswahl der japanischen Säuberungskomitee-Mitglieder keineswegs immer sorgfältig genug verfahren worden war ${ }^{10}$.

Die wachsende amerikanische Kritik an einer allzu weichen und einfühlsamen Säuberungspraxis schlug sich im August 1946 in der Forderung des SCAP an die japanische Regierung nieder, einen Plan für die Erweiterung des purge in den Bereich der Lokalpolitik, der Medien und der Wirtschaft hinein vorzulegen. Die durch Kaiserliche Erlasse am 4. Januar 1947, also am Jahrestag der ersten Säuberungsdirektive, beginnende zweite Welle der Entmilitarisierung brachte vor allem für die Betroffenengruppe der „sonstigen Militaristen und Ultranationalisten“ (G) eine Reihe von Präzisierungen und Verschärfungen, die indes auch die Grundparadoxie der Säuberungspolitik noch schärfer ins Auge fallen ließen: die Ambivalenz zwischen einem in erster Linie vergangenheitsorientierten punitiven und einem auf die Zukunft der japanischen Demokratie gerichteten präventiven Ansatz ${ }^{11}$. Einflußreiche Personen aus dem öffentlichen Leben auszuschalten, weil sie den Krieg mit zu verantworten hatten, war eine Sache, es zu tun, weil sie eine potentielle Gefahr für die neue Demokratie darstellten, bedeutete eine schwer in den Griff zu bekommende Ausweitung der Säuberungspraxis. So gab es gerade in der Beamtenschaft Personen, die Gegner des aggressiven Expansionskurses gewesen waren, die aber jetzt weiterhin erzkonservativ am Primat des Staates über das Individuum festhielten, während an sich unverdächtige Parlamentarier aus der Zeit vor 1937 die ultranationalistische Politik später oft genug unterstützt hatten.

Ein Weg aus dem Säuberungsdilemma wäre es gerade auf der lokalen Politikebene gewesen, klar zu legen, daß das alte Führungspersonal nicht in erster Linie wegen seiner Kriegsschuld entfernt wurde, sondern einzig und allein, um Platz für neue Eliten zu schaffen. Statt dessen aber galt es den schwierigen Nachweis zu führen, daß Tausende Bürgermeister, Landräte oder örtliche Funktionäre des Bundes zur Förderung der Kaiserlichen Herrschaft eine führende Rolle „bei der Täuschung und Irreführung des japanischen Volkes" gespielt hatten ${ }^{12}$. Der Widerstand der regierenden Konservativen gegen die Erweiterung der Säuberung in die Fläche mußte um so heftiger ausfallen, als ihre Parteien infolge der ersten Welle bereits einige wichtige nationale Persönlichkeiten verloren und sich dann auf ihre ländlichen Bastionen gestützt hatten, um die Lücken wieder zu schließen. Ohne

10 Ebd., S. 122-127.

11 Letzterer war vor allem MacArthur ein Anliegen gewesen. Vgl. Baerwald, The Purge, S. 9 f., 25.

12 Ebd., S. 26. 
die Unterstützung der 1946 zahlreich in das Unterhaus eingezogenen Kommunalpolitiker wäre zudem die Verabschiedung eines neuen Wahlgesetzes fraglich geworden, das den konservativen Parteien schon bei den bevorstehenden neuerlichen Wahlen Vorteile verschaffen sollte. ${ }^{13}$

So war auch der zweiten Säuberungswelle aufgrund der dilatorischen Haltung der japanischen Administration nur ein relativer Erfolg beschieden. In den Wochen vor den General elections im April $1947 \mathrm{zu}$ den beiden Häusern des Parlaments und den kommunalen Vertretungskörperschaften fielen dem screening von 3246 überprüften Unterhauskandidaten 137 Personen zum Opfer, von 1406 Oberhauskandidaten 31, von 451 präsumtiven Präfekten 20 und von den 43 Bürgermeisterkandidaten in fünf Großstädten nur ein Politiker. Nicht höher lagen die Säuberungsquoten auch bei den weiteren mehr als 100000 durchleuchteten Kommunalpolitikern in den kleineren Gemeinden ${ }^{14}$. Im Mai 1948 hatte der purge bereits seine „Hochwassermarke" erreicht, um sich in seiner letzen Phase (1948-1952) vom Sommer 1948 an zunehmend in eine „holding operation“ 15 zu verwandeln, während gleichzeitig von den Appeal boards ( schon ab März 1947) ganze Säuberungsgruppen rehabilitiert wurden und statt dessen im einsetzenden Red Purge der Jahre 1949 bis 1951 über 20000 Kommunisten bzw. Sympathisanten ihren Arbeitsplatz in Regierung, Massenmedien oder Privatwirtschaft verloren ${ }^{16}$. Die Zahl ist um so bemerkenswerter, wenn man sie mit der nicht sehr hohen Schlußbilanz der Entmilitarisierung selbst vergleicht, deren Gesamtzahl sich auf etwas über 210000 purgees belief, wovon freilich über drei Viertel Soldaten waren; knapp 35000 (16,5\%) zählten zur politischen Elite und nur wenige Tausend zur administrativen, ultranationalistischen, wirtschaftlichen und massenmedialen Führungsschicht ${ }^{17}$. Hinzu kamen 6000 ehemalige Angehörige der Gedankenpolizei und einer weiteren Spezialpolizeieinheit, denen durch eine eigene Civil-Liberties-Direktive des SCAP vom Oktober 1945 zumindest der Zugang zu Tätigkeiten im Bereich von Justiz und innerer Sicherheit versperrt wurde. In der Lehrerschaft, wo 115000 Personen bereits den „präventiven Rückzug“ gewählt hatten, sahen sich bis zum Abschluß der speziellen Überprüfungsmaßnahmen im Erziehungsbereich Ende April 1949 über dreitausend Pädagogen vom Dienst suspendiert ${ }^{18}$. Zwei weitere Zahlen illustrieren die beschränkte Reichweite der Säuberung und die rasche Rückkehr der Gesäuberten ins öffentliche Leben vielleicht am anschaulichsten: Bei den Wahlen zum Abgeordnetenhaus im

13 Ebd., S. 29.

14 Dokument 1, in: Benz, Amerikanische Besatzungsherrschaft, S. 306. Eine vertiefte Darstellung der zweiten Säuberungswelle bietet auch: Political Reorientation of Japan. September 1945 to September 1948, hg. vom General Headquarter, S. 37 ff.

15 Baerwald, The Purge, S. 78.

16 Zum Red Purge vgl. Nishi, Unconditional Democracy, S. $242 \mathrm{ff}$.

17 Baerwald, The Purge, S. 80.

18 Rosenzwcig, Erziehung, S. 129. 
Oktober 1952 eroberten ehemalige purgees 139 von 466 Sitzen; und zwei Jahre später gehörten dem Kabinett Hatoyama - ihn eingeschlossen - 12 Gesäuberte an ${ }^{19}$.

In einer frühen Vergleichsstudie über die „künstlichen“, d.h. qua Besatzungsmacht durchgeführten Revolutionen in Deutschland und Japan nach 1945, wo anders als etwa in Italien nicht von der Fiktion einer bereits stattgefundenen demokratischen Umwälzung ausgegangen werden konnte, sondern die politisch unerwünschte alte Führung von den Siegermächten durch eine neue Elite zu ersetzen war ${ }^{20}$, hat Montgomery die mechanische Dimension der japanischen Entmilitarisierung zu erklären versucht: Diese sei - anders als die Entnazifizierung in Deutschland - nicht „under a shower of moralistic words and grandiose objectives" durchgeführt worden, sondern eher „in a realistic and matter-of-fact way“ 21 . Doch unverkennbar nahm auch die Entnazifizierung in der US-Zone - gerade wegen ihrer im Kern moralischen und nicht pragmatischen Zielrichtung - letztlich mechanische Züge an.

Die Amerikaner inaugurierten in Deutschland bereits im April 1945 mit der Direktive JCS 1067 eine gründliche Säuberung des öffentlichen Lebens, der Wirtschaft und des Erziehungswesens, indem sie die mehr als nur nominell in der NSDAP tätigen Parteigenossen (etwa das Korps der Politischen Leiter, Angehörige von SS und Gestapo) sowie all jene, die Nazismus und Militarismus aktiv unterstützt hatten, aus staatlichen Stellen, aber auch aus wichtigen Positionen in halböffentlichen und privaten Unternehmungen entfernten und wegsperrten. Volle Internierungslager und leere Ämter waren die Folge. Keineswegs nur der höhere Dienst in den Reichs- und Landesbehörden wurde von den Entlassungen betroffen, sondern auch zahlreiche kleinere Beamte in regionalen und lokalen Dienststellen. Die Bürgermeister sahen sich in manchen Landkreisen samt und sonders ihres Amtes enthoben; und in der bayerischen Lehrerschaft verlor etwa jeder zweite seinen Arbeitsplatz. Als die allzu große Rigorosität und Pauschalität der Massenentlassungen und ihre absehbar desaströsen Folgen für den Wiederaufbau immer deutlicher hervortraten, wurde die erste, mehr oder weniger wilde Säuberungsphase abgebrochen und die delikate Prozedur der Entnazifizierung mit dem „Gesetz zur Befreiung von Nationalsozialismus und Militarismus“ am 5. März 1946 in die Hände der neugebildeten deutschen Behörden gelegt. Auch dem neuen Verfahren eigneten wiederum gravierende Mängel, verfolgte es doch die Herkules-Aufgabe, die Vergangenheit sämtlicher erwachsener Deutscher nötigenfalls in Spruchkammerverfahren zu durchleuchten, woraus in den Westzonen über dreieinhalb Millionen zu verhandelnde Fälle resultierten ${ }^{22}$.

19 Harrics, Sheathing the Sword, S. 197.

20 Montgomery, Forced to be Free, S. 17.

21 Ebd., S. 30.

22 Aus der umfangreichen Literatur zur Entnazifizierung genannt sei als Überblick Vollnhals, 
Auch wenn in Deutschland vor allem die personelle Erblast einer untergegangenen und verbotenen Massenpartei zu bewältigen war, während sich die Säuberung in Japan in allererster Linie gegen das Militär richtete und erst in zweiter Hinsicht die Mitglieder der weiterbestehenden Parteien ins Visier genommen wurden, waren in der Praxis von Entnazifizierung und Entmilitarisierung ähnliche Phänomene zu beobachten - nicht zuletzt Versuche, die Säuberung zur Bekämpfung aktueller politischer Gegner zu mißbrauchen. Man denke nur an die vom konservativen Kabinett veranlaßte Schwächung der oppositionellen Minshutô (Demokratische Partei) unmittelbar vor den Wahlen vom April 194723 oder die Erfassung des Vizepräsidenten des Oberhauses Matsumoto Jiichirô, eines bekannten buraku24-Politikers, auf einer Säuberungsliste mit Namen von ,in den Kriegsverbrecherprozessen verantwortlich gemachten“ Anführern der „japanischen Kriegsbemühungen" 25 . Tatsächlich hatte Matsumoto - nach Kriegsende - ein ganz anderes Sakrileg begangen: nämlich bei der Parlamentseröffnung $1948 \mathrm{dem}$ Kaiser den traditionellen Kotau zu verweigern. In Westdeutschland erregten vor allem der gescheiterte Versuch, den CSU-Gründungsvorsitzenden Josef Müller per Spruchkammerverfahren mundtot zu machen ${ }^{26}$, oder mehr noch die Affäre „Maier gegen Maier“, öffentliche Aufmerksamkeit, als der Lizenzträger der Stuttgarter Zeitung und Kläger an der dortigen Spruchkammer, Franz Karl Maier, in dem Theodor Heuss einen „Robespierre von Ochsenhausen“ erblickte, seine aktuellen publizistischen Attacken gegen den liberalen württembergischen Ministerpräsidenten Reinhold Maier mittels Entnazifizierung fortsetzte und ihn wegen seiner Zustimmung zum Ermächtigungsgesetz 1933 - letztlich ohne Erfolg - zur Anklage brachte27.

Vergleichbare Säuberungsmängel in Japan und Deutschland dürfen über eines nicht hinwegtäuschen: Bei der Entmilitarisierung wurde konsequenterweise mit der Bestrafung der am schwersten Belasteten begonnen, während bei der Entnazifizierung infolge der Prozeßflut pragmatische Gründe dafür sprachen, die schweren Fälle zurückzustellen und die einfachen gleich zu Beginn abzuhandeln, schon um den Betroffenen eine möglichst rasche Rückkehr ins Berufsleben zu ermöglichen. Dies führte freilich dazu, daß wegen des abrupten Abwürgens des Säuberungsprozesses 1947/48 im eskalierenden Kalten Krieg viele der „wahren Schuldigen“ in den Genuß einer

Entnazifizicrung, sowie Regionalstudien älteren bzw. neucren Datums: Nicthammer, Entnazifizierung in Bayern, Schuster, Die Entnazifizierung in Hessen.

23 Quigley/Turner, The New Japan, S. $108 \mathrm{f}$.

24 Nachfahren der einst u.a. aus religiösen Gründen wegen ihres „schmutzigen“ Berufs (Abdecker, Gerber etc.) aus der Gesellschaft Ausgestoßenen, die auch nach ihrer formal-juristischen Gleichstellung 1871 in ghettoähnlichen Siedlungen ein isoliertes Leben führten.

25 De Vos, Japan's Outcasts, S. 15.

26 Hettler, Josef Müller, S. $280 \mathrm{ff}$.

27 Hierzu die umfassende Darstellung von Matz, Reinhold Maier, S. 276-304. 
milderen Entnazifizierungspraxis kamen ${ }^{28}$ und sich in der Bevölkerung der später vielzitierte Eindruck einstellte: „Die Kleinen hängt man, die Großen läßt man laufen“. Zur immer größer werdenden Skepsis der Deutschen gegen die personelle „Vergangenheitsbewältigung“ trug diese Entwicklung ebenso bei wie die "Geburtsfehler" des Befreiungsgesetzes, d.h. die von vornherein weite Überdehnung des abzuurteilenden Personenkreises und die „Verlagerung politischer Säuberungsvorgaben auf die entpolitisierte Ebene" eines gerichtsähnlichen Verfahrens ${ }^{29}$. Immerhin wurde dieses mit erheblichem organisatorischen Aufwand in 545 Spruchkammern von 22000 meist Laienmitgliedern betrieben.

So hatte in der amerikanischen Zone Deutschlands jeder Prüfer statistisch gesehen nur 16,47 Personen zu durchleuchten, während es in Japan 769,6 waren. Zwischen dem 20. Februar und dem 10. März 1947 überprüfte das nationale Screening board täglich allein 900 Kandidaten für die kommunalen Parlamente. Waren auf der Insel im fernen Osten insgesamt 3,2\% der Bevölkerung überprüft worden, so galt dies in der US-Zone für $21,7 \%$, und auch amtsenthoben wurden in Westdeutschland prozentual etwa zehn mal mehr Personen ${ }^{30}$. Den gut 200000 gesäuberten Japanern standen nach Berechnungen von Montgomery allein in der amerikanischen Besatzungszone Deutschlands 418307 Entnazifizierte gegenüber. Tatsächlich verhängten die Spruchkammern in 600000 Fällen Strafen, wobei 500000 Betroffene mit einer Geldstrafe oder kleineren Sühneleistung davonkamen. Aus führenden Positionen im öffentlichen Leben ausgeschlossen wurden im Sinne des Befreiungsgesetzes vor allem die etwa 1600 Hauptschuldigen und 22000 „Belasteten", während das Gros der 150000 Minderbelasteten lediglich eine Bewährungszeit zu bestehen hatte. Eine statistische Erhebung im April 1950 bezifferte die Zahl der nach dem 8. Mai 1945 ausgeschiedenen „und bisher nicht oder nicht ihrer früheren Stellung entsprechend" verwendeten entnazifizierten Beamten in Westdeutschland insgesamt - vermutlich zu niedrig auf 55000; hinzu kamen mindestens 150000 ehemalige Berufssoldaten und eine ähnlich große Zahl Heimatvertriebener und sonstiger Versorgungsberechtigter ${ }^{31}$.

Der zentrale, langfristig folgenreiche Unterschied in der Eröffnungsphase der „Vergangenheitsbewältigung“ resultierte im wesentlichen aus zwei gegensätzlichen Entwicklungen: Zum einen aus der ungleich größeren Schärfe der Maßnahmen im besetzten Deutschland mit der zeitweiligen Internierung von über 200000 NS-Aktivisten allein in den Westzonen im Zuge des automatic arrest. Hierfür gab es in Japan überhaupt keine Entsprechung,

28 So auch die Kritik des bayerischen SPD-Ministerpräsidenten Wilhelm Hoegner, zit. nach Kritzer, Wilhelm Hoegner, S. 245.

29 Vollnhals, Entnazifizierung, S. 55.

30 Montgomery, Forced to be Free, S. $26 \mathrm{ff}$.

31 Reichel, Vergangenheitsbewältigung, S. 112. Vgl. auch Wengst, Beamtentum, S. 172. 
weil die Besatzungsmacht das innere Gefahrenpotential wegen des Fehlens einer faschistischen Staatspartei mit Bewegungscharakter ganz anders einschätzte. Zum anderen hatten auch viele kleine NS-Pg's, die selbst keine Bekanntschaft mit Internierungslagern machten, nicht nur oft Jahre warten müssen, bis sie einen entlastenden Bescheid ausgestellt bekamen und wieder dem alten Beruf nachgehen konnten, sondern waren sie immerhin millionenfach zur Anklage gebracht worden und hatten buchstäblich einen Denkzettel erhalten. Dessen Mischung aus „fühlbarer Strafe und großzügiger Gnade" verhinderte, daß ein Heer von Entnazifizierungsgeschädigten entstand, das den Aufbau der Bundesrepublik übermäßig erschwert hätte, förderte aber wesentlich „das kritische Nachdenken über antidemokratische Experimente und Ideologien" 32 .

Nun fiel dieser Effekt in Japan, anders als man aufgrund der vorliegenden Zahlen quantifizieren könnte, sicher nicht zehnmal geringer aus als in Deutschland. Das Bewußtsein von der eigenen Kompromittierung während des vergangenen politischen Systems und die Bereitschaft, sich auch öffentlich mit dessen Verbrechen auseinanderzusetzen, ist aber von der Entmilitarisierung offensichtlich doch weniger gefördert worden als von der Entnazifizierung.

Dies galt in besonderem Maße für die Beamtenschaft, die in Deutschland selbst in ihren einfachen Rängen zumindest in Form der Massenentlassungen von 1945 hunderttausendfach besagten „Denkzettel“ erhielt, während in ganz Japan überhaupt nur 830 Verwaltungsbeamte infolge der Säuberung ihren Arbeitsplatz verloren. Die gesamtgesellschaftliche Bedeutung dieses Umstands dürfte kaum zu überschätzen sein, weil damit der Einfluß einer als Diener des Tennô ohnehin sehr angesehenen - Bevölkerungsgruppe konserviert wurde, die - marxistisch gewendet - „eng mit dem militärischindustriellen Komplex und dem japanischen Militarismus und Expansionismus verwoben“ war und von der es infolgedessen schon wenige Jahre nach dem Krieg mit einigem Recht hieß, sie sei mehr als jede andere Gruppe „in der japanischen Vergangenheit vor der Kapitulation verwurzelt ${ }^{\text {“ } 33}$.

Neuerdings in eine Schlüsselstellung rückten zunächst vor allem die höheren Beamten des Auswärtigen Dienstes ein, weil SCAP glaubte, für das „Zentrale Verbindungsbüro“ auf die Dienste der sprachgewandten Männer nicht verzichten zu können ${ }^{34}$. Obendrein wurde die gesamte Beamtenschaft durch die vergleichsweise strengere Entmilitarisierung der politischen Parteien indirekt gestärkt, weil aus dem Kreis der Staatsdiener nun etliche, bald führende Abgeordnete hervorgingen, die von den in die Reihen der alten Berufspolitiker gerissenen Lücken profitierten. Auch wenn das Gros der

32 Woller, Gesellschaft und Politik, S. 163.

33 Halliday, Japan unter amerikanischer Besatzung, S. 119.

34 Kawai, Japan's American Interlude, S. 198. 
entnazifizierten deutschen Beamtenschaft nach seiner raschen Wiedereinstellung ebenfalls bald wieder mit beträchtlichem Erfolg auf den „wohlerworbenen " materiellen Rechten beharrte, zahlreich in die Parlamente einrückte und ähnlich wie in Japan eine grundlegende Reform des öffentlichen Dienstes verhinderte, schuf die Erfahrung des totalen Zusammenbruchs 1945 doch unverkennbar günstigere Voraussetzungen für eine Schwächung des Prestiges, des Korpsgeists und der überkommenen nationalistischen Blickverengungen einer Berufsgruppe, die wegen der staatlich-bürokratischen Regelungsdichte des Alltags Stil und Inhalt der neuen Demokratie nicht unwesentlich mitprägte ${ }^{35}$.

Dies galt gerade auch für das neue Auswärtige Amt (AA). Dessen Beamte rückten Anfang der 1950er Jahre mit am stärksten in das Blickfeld einer vergangenheitskritischen Öffentlichkeit, die freilich nicht immer dem „Irrtum der Gleichsetzung von ehemaligen Parteigenossen und Nationalsozialisten" 36 entging. Da sich Bundeskanzler Adenauer in dem zentralen Feld der Außenpolitik besonders auf die Professionalität der traditionellen Funktionseliten angewiesen fühlte, handelte er nach einer Devise seiner Kölner Heimat: „Man schüttet kein dreckiges Wasser aus, wenn man kein reines

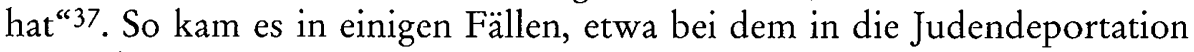
von Belgien nach Auschwitz verstrickt gewesenen und vom AA wieder eingestellten Werner von Bargen, zu skandalösen personellen Kontinuitäten. Die besonders scharf von den Kommunisten im Bundestag verfochtene These, „die Ribbentropler, die in der Nazizeit Hitlers Außenpolitik im Ausland ... durchgeführt" 38 hätten, würden im Zuge einer „Totalrestauration der Wilhelmstraße“ jetzt das auf der Linie Hitlers liegende außenpolitische Konzept Adenauers umsetzen, war jedoch weit überzogen. Gerade Diplomaten aus dem engsten Umfelds Ribbentrops und solche mit früher Bindung an die SS wurden in der Regel nicht mehr übernommen. Darüber hinaus wurde auch die - Adenauer stets suspekte - traditionelle Dominanz von Beamten preußisch-protestantischer Herkunft zu Gunsten südwestdeutscher Katholiken zurückgedrängt ${ }^{39}$.

Nicht einfach als mißlungen kann auch die personelle „Vergangenheitsbewältigung“ im Bereich der Wirtschaft gelten. Zum einen waren die Unter-

35 Vgl. hierzu auch Kocka, Kontinuitäten und Wandlungen, vor allem S. 30-34, der stärker die Kontinuitäten im „Restaurationsprozeß“ nach 1945 betont hat, sowie grundlegend vor allem Wengst, Beamtentum.

36 So Staatssekretär Walter Hallstein im Blick auf die voreiliger Generalisierung entspringende Empörung über den 65\%-Anteil ehemaliger Pg's an den leitenden Beamten im neuen Auswärtigen Amt. Frankfurter Allgemeine Zeitung, 27. März 1952.

37 Adenauer, Teegespräche 1950-1954, S. 245.

38 Verhandlungen des Deutschen Bundestages, 22. Oktober 1952, S. $10740 \mathrm{ff}$.

39 Vgl. hierzu dic Ergebnisse der Studie von Döscher, Verschworene Gesellschaft, S. $311 \mathrm{ff}$., deren Wert auch durch das ideologische Geleitwort von Manfred Steinkühler kaum gemindert wird. 
nehmen von der Militärregierung 1945 verpflichtet worden, NSDAP-Mitglieder unverzüglich aus beaufsichtigenden oder leitenden Stellungen zu entfernen. Selbst kleinen Geschäftsleuten und Handwerksmeistern drohte die Einsetzung von Treuhändern. Unter Kontrolle befanden sich allein in Bayern 1947 noch mehr als 1000 Industriebetriebe und 4000 Handels- und Dienstleistungsfirmen, die erst ab Mitte 1948 wieder an die zwischenzeitlich entnazifizierten Eigentümer zurückgegeben wurden. Besonders „große Fische" wie etwa die 1948 in Nürnberg als Hauptkriegsverbrecher verurteilten Direktoren des Chemiekonzerns IG-Farben mußten mehrjährige Haftstrafen abbüßen. Zwar rückten sie später oft genug wieder in wichtige Positionen der deutschen Wirtschaft ein, doch blieb ihre zumindest zeitweilige Ausschaltung nicht folgenlos. Vielmehr wurde der Elite der deutschen Industrie während der Jahre der Internierung und Deklassierung überhaupt erst richtig bewußt, daß die Alliierten auch ihrer Berufsgruppe eine Mitschuld für das NS-Regime zumaßen. Dieses Bewußtsein mußte zwar wieder getrübt werden, wenn etwa einem früheren Direktor der Leunawerke, der sich der Versklavung von Fremdarbeitern schuldig gemacht hatte, für Leistungen in der Zeit des Wiederaufbaus das große Verdienstkreuz der Bundesrepublik verliehen wurde. Solche Fälle dürfen aber nicht darüber hinwegtäuschen, daß zumindest diejenigen Wirtschaftskapitäne, die „im Dienste der nationalsozialistischen Parteiwirtschaft Standeskomment und Belange der Privatindustrie verletzt“ hatten, nach 1945 „als schwarze Schafe für immer aus der Gnade ihrer Gruppe fielen“ und „zur Entlastung der manchmal nur geringfügig weniger Kompromittierten“ ausgegrenzt blieben $^{40}$.

Die auch in Deutschland gegen Großbanken, Montan- und Chemieindustrie gerichtete Entflechtungspolitik der Amerikaner gewann in Japan wegen der Existenz der zaibatsu besondere Bedeutung. Bei diesen um die Wende zum 20. Jahrhundert bzw. beim Aufbau der Schwerindustrie in der Mandschurei entstandenen „Finanzcliquen“ handelte es sich um teils gigantische Familientrusts, die Industrie-, Handels- und Bankkapital in Hunderten von Unternehmen mit teils über einer Million Mitarbeitern vereinigten. $\mathrm{Da}$ auf dem Bündnis der größten Familienkonzerne mit den leitenden Staatsbeamten und der Generalität die politische Macht Japans auch während des Kriegsregimes beruht hatte, war für die amerikanischen Besatzer die Zerschlagung der zaibatsu ein vordringliches Ziel im Rahmen der Entmilitarisierung. Während der Ende 1945 einsetzenden Auflösung der größten Konzerne Mitsui, Mitsubishi, Sumitomo und Yasuda sowie weiterer 18

40 Henke, Die Trennung vom Nationalsozialismus, S. 59, 63. Vgl. auch Woller, Politik und Gesellschaft, S. 253. 
zaibatsu mit 83 Holding- und um die 4000 Tochtergesellschaften mußten auch zahlreiche leitende Angestellte ihren Posten räumen ${ }^{41}$.

Die während der ultranationalistischen Periode aufgestiegenen und als Kollaborateure des Militarismus gesäuberten Manager, die durch das Berufsverbot zwischen 1946 und 1951 von der unmittelbaren Geschäftsführung ausgeschlossen blieben, wurden allerdings ab 1952 wieder unverzüglich in leitende Positionen ihrer Unternehmen eingesetzt. Auf der Basis des noch während der Besatzungszeit infolge der großen Wende von 1948 wieder gelockerten Monopolverbotsgesetzes entstanden nun „in liberalkaschierter Form" 42 Großbanken mit angeschlossenen Großhandelshäusern, in denen es den vielfach verschwägerten oder anderweitig verbundenen Gliedern der alten zaibatsu-Familien niemand verwehren konnte, sich auch ohne formale Rechtskonstruktion informelle Kooperationsstrukturen (keiretsu) zu schaffen. Wohl in keinem anderen Bereich, so das Fazit von Meirion und Susie Harries, sei das Scheitern der Reformkräfte innerhalb der amerikanischen Besatzungsmacht offenkundiger gewesen "than in the sabotage of the deconcentration programme in favour of the economic ,crankup' "43 Allerdings stand die von den keiretsu fortgeführte zaibatsu-Tradition jetzt doch in vermehrter Konkurrenz zu einer liberalen Unternehmerrichtung, die in der Zeit nach dem Ersten Weltkrieg sozialisiert worden war und durch die Ausschaltung der Senioren aus den zaibatsu-Konzernen nach 1945 ihre Position hatte ausbauen können ${ }^{44}$.

Trotz unübersehbarer Parallelen zwischen der Säuberung der japanischen und der deutschen Wirtschaft waren die Dimensionen des Vorgangs doch sehr unterschiedlich. Fielen in ganz Japan nur knapp 2000 Personen dem purge ${ }^{45}$ zum Opfer, so schieden allein im amerikanisch besetzten GroßHessen infolge des Befreiungsgesetzes fast ebenso viele Betriebsleiter und leitende Angestellte aus ihrer Stellung aus ${ }^{46}$. Für die Zahl der in den Westzonen verhängten Berufsverbote gab es in Japan ebensowenig eine Entsprechung wie für die Schlüsselerfahrung von Verhaftung und Internierung. Dies gilt schließlich in besonderem Maße für die Tatsache, daß keiner der großen zaibatsu-Führer in Tokio spektakulär zur Anklage gebracht wurde, während sich 1947 bei einem der großen Nürnberger Nachfolgeprozesse zumindest 36 Direktoren und Firmenleiter des Flick-Konzerns, der IGFarben und von Krupp gleichsam stellvertretend für die deutsche Wirtschaft vor ein internationales Gericht gestellt sahen. Damit dokumentierten

41 R. Hartmann, Geschichte des modernen Japan, S. 217.

42 Zahl, Die politische Elite, S. 191.

43 Harries, Sheathing the sword, S. 204.

44 Zahl, Die politische Elite, S. $189 \mathrm{ff}$.

45 Zur Säuberungsstatistik beim „ökonomischen Personal“ siehe Baerwald, The Purge, S. $91 \mathrm{f}$.

46 Bericht des Statistischen Landesamtes über die Entnazifizierung der hessischen Wirtschaft bis zum 31. Mai 1946 (Auszug), in: Vollnhals, Entnazifizierung, S. 161. 
die Amerikaner vor aller Welt, daß die deutschen Wirtschaftsführer nicht nur unter dem Zwang der Verhältnisse, sondern auch aus eigenem Expansions- und Profitinteresse selbständig Krieg und Verbrechen unterstützt hat$\operatorname{ten}^{47}$.

Die doch sehr unterschiedlichen Ergebnisse von Entmilitarisierung und Entnazifizierung wurzelten letztlich schon im differierenden Ansatz der amerikanischen Planungsstäbe für die Besetzung Deutschlands und Japans. Die meisten US-Deutschlandexperten waren deutschstämmige Amerikaner oder Emigranten, die „mit Deutschland hart ins Gericht" gingen und sich in geistes- und erziehungswissenschaftlichen sowie psychologischen Zirkeln fragten, ob diese Nation "vielleicht schon immer krank gewesen sei“48. Zwei Denkschulen sind dabei zu unterscheiden. Die „Maximalisten“ deuteten den Nationalsozialismus als Folge einer pathologisch deformierten politischen Kollektivmentalität der Deutschen, hielten politische Säuberungen demzufolge für unzureichend und forderten die Auflösung „autoritärer“ politischer, wirtschaftlicher und gesellschaftlicher Strukturen, damit Deutschland nie wieder als kapitalistische Großmacht in der Lage wäre, einen Krieg zu führen ${ }^{49}$. Kein geringerer als der unter Roosevelt ausgesprochen einflußreiche US-Finanzministers Henry Morgenthau zählte zu den prominentesten Anhängern dieser Richtung. Die „Minimalisten“ dagegen mit Schützenhilfe von Kriegsminister Henry Stimson und Justizminister Francis Biddle - plädierten dafür, die Entnazifizierung auf die oberste Führungsschicht der Nationalsozialisten zu beschränken und möglichst rasch das politische System der Weimarer Republik wiederherzustellen. So zerstritten die amerikanischen Deutschlandplaner in einzelnen Fragen auch waren, so sehr nahmen sie „das Schuldeingeständnis der Deutschen zunächst persönlich wichtig“ 50 .

Die US-Japanplaner dagegen ließen „den Aspekt der japanischen historischen und moralischen Schuld in all ihren Entwürfen unberücksichtigt." "51 Und zwar offensichtlich deshalb, weil es sich bei ihnen um Personen handelte, die über positive persönliche Bindungen an Japan verfügten. Sie waren darauf bedacht, ein günstiges Bild des Landes zu vermitteln, hatten indes „weder einen ausreichenden Abstand noch eine verwurzelte Nähe zu Japan" 52 , um sich kritisch genug mit Fragen der Kriegsschuld zu befassen. Zudem hatte die Gruppe der - weißen angloamerikanischen - Japanexperten einen ausgesprochen homogenen Charakter, weil japanischstämmige Amerikaner sich entweder in Internierungslagern befanden oder zumindest

$47 \mathrm{Vgl}$. Erker, Einleitung: Industrie-Eliten im 20. Jahrhundert, S. 9.

48 Hentschke, Demokratisierung als Ziel, S. 125.

49 Ebd., S. 69 f.

50 Ebd., S. 125.

51 Ebd.

52 Ebd., S. 126. 
aus Gründen rassischer Diskriminierung nicht herangezogen wurden. So war das Klientel derer, auf die seitens der US-Regierung für die Planungsaufgabe zurückgegriffen werden konnte, von vornherein sehr überschaubar und bot keine große Auswahl. Zwar mußten die Japanplaner zeitweilig erschreckt zur Kenntnis nehmen, daß Morgenthau ihre Konzepte einer indirekten Okkupation des Inselstaates durchkreuzen und eine ähnlich harte Politik wie für Deutschland durchsetzen wollte, doch gelang es ihnen, diese Strategie zu vereiteln. Zu Hilfe kam ihnen dabei schließlich auch das Ableben Roosevelts, unter dessen Nachfolger Truman Morgenthau nicht einmal mehr den Versuch unternahm, japanpolitisch zu intervenieren. Nach dem Tod von Roosevelt stand, so ist gesagt worden, „der Japan-Lobby nichts mehr im Weg“"53 - außer, so wäre hinzuzufügen, jenen, durch die politische Entwicklung in Ostasien indes bald widerlegten Kräften, die zunächst noch auf die (national-)chinesische Karte setzten.

Gegen allzu fundamentalistische Kritik an den Ergebnissen der Entmilitarisierung in Japan hat Hans H. Baerwald mit einigem Grund darauf hingewiesen, daß dem Erreichen demokratischer Ziele mit autoritären Methoden von vornherein Grenzen gesetzt seien ${ }^{54}$. Die deutlich weiter reichende Entnazifizierung in Deutschland liefert aber ein Gegenargument. Trotz unterschiedlicher Ausgangsbedingungen wäre auch in Japan mehr möglich gewesen, wenn die USA - und vor allem auch der Stab MacArthurs - nur den politischen Willen dazu gehabt hätten. Denn in keinem anderen Bereich der „Vergangenheitsbewältigung" kam es so wenig auf die erinnerungskulturellen Dispositionen des betroffenen Landes und so sehr auf die Direktiven der Besatzungsmacht an wie bei der personellen Säuberung.

53 Ebd., S. 115.

54 Baerwald, The Purge in Occupied Japan, S. 197. 